\title{
Evaluation of the geriatric patients prescription for inappropriate medications frequency at Larkana Sindh Hospital in Pakistan
}

\author{
Altaf A. Mangi ${ }^{\mathrm{a}}$, Mohamed Anwar Hammad ${ }^{\mathrm{b}, *}$, Haroon Khan ${ }^{\mathrm{a}}$, Shumaila P. Arain ${ }^{\mathrm{c}}$, \\ Muhammad A. Shahzad ${ }^{\mathrm{a}}$, Eshwa Dar ${ }^{\mathrm{d}}$, Anila Alam ${ }^{\mathrm{e}}$, Ahmed H.A. Hassanein ${ }^{\mathrm{f}}$ \\ ${ }^{\text {a }}$ Faculty of Pharmacy, Gomal University, Dera Ismail Khan, Pakistan \\ ${ }^{\mathrm{b}}$ Department of Clinical Pharmacy, Faculty of Pharmacy, Sinai University, Arish, North Sinai, 45615, Egypt \\ ${ }^{\mathrm{c}}$ Faculty of Pharmacy, University of Sindh, Jamshoro, Pakistan \\ ${ }^{\mathrm{d}}$ Department of Clinical Pharmacy, Faculty of Pharmacy, The University of Lahore, Punjab, Pakistan \\ e Sardar Bahadur Khan Women Univesity, Quetta, Balochistan, Pakistan \\ ${ }^{\mathrm{f}}$ Department of Clinical Pharmacy, Faculty of Pharmacy, Shaqra University, Shaqra, Saudi Arabia
}

\section{A R T I C L E I N F O}

\section{Keywords:}

Beers criteria

Geriatrics patients

Inappropriate medication

Medication error

Polypharmacy prescription

\begin{abstract}
A B S T R A C T
Background: Aging is the irreversible change in the human physiology which occurs with the passage of time and it becomes very much critical to recommend drugs among the geriatrics patients because their body response is quite different.

Objectives: The purpose of the current study is to assess and evaluate the potentially inappropriate medications among the geriatric patients at the tertiary care Hospital of Larkana Sindh which is the biggest Hospital in the Northern Part of Sindh.

Methods: The descriptive cross-sectional study was conducted which included the 500 patients aged more than 60 years. The data was collected from the patients' case charts and the questionnaire distributed among the patients. BEERS' criteria was used to detect the frequency of chronic disorders in geriatric patients and appraise the polypharmacy.

Results: The male patients were $42.6 \%$ and the female patients were $57.4 \%$. The inappropriate medication use (IMU) was observed among $22.6 \%$ patients. Inappropriate antibiotics were seen in $35.39 \%$, Benzodiazepine $28.32 \%$, non-steroidal anti-inflammatory drugs (NSAIDs) $15.04 \%$, cardiovascular system (CVS) drugs $12.39 \%$ of patients. Parenteral route of administration was observed in $70.04 \%$ of patients.

Conclusion: Polypharmacy is highly prevalent in the prescriptions of geriatrics patients, due to certain reasons like a chronic disease. Clinical Pharmacist can be better placed to provide his professional activities in this context.
\end{abstract}

\section{Introduction}

Aging is the natural phenomenon which is experienced over the time and its alteration in the organ function irrespective to any injury or malady. ${ }^{1}$ Normal aging and the malady concerned aging are two different things.Normal aging is the normal deteriorative process of tissue decay which is observed when the human being live long enough and the certain changes are being observed like, decreased bone mass, Arthritis, dementia, stroke and the congestive heart failure (CHF). ${ }^{2}$ Aging is of the critical stage of life where especially the use of medication needs more care and sound knowledge with skills because aged body responds medicine very separately comparatively to the young body. ${ }^{3}$

Especially the vital organ disease in the geriatric patients like disease of kidneys, liver, central nervous system (CNS) and heart are majorly responsible for the overdose and the side effects of the medications. and overlooking these alterations in the treatment or management leads to the irrational use of medications. ${ }^{4,5}$ Several studies have been done in the developing and the developed countries which depicts that the irrational and inappropriate medication usage is the global phenomenon which requires the serious attention. ${ }^{6,7}$

The definition of the rational drug therapy as per World Health Organization (WHO) is the right medicine for the right patient at right

\footnotetext{
* Corresponding author.

E-mail addresses: pharmacistsindh41@gmail.com (A.A. Mangi), m_anwaaar@hotmail.com (M.A. Hammad), haroonscholar@gmail.com (H. Khan), gnabi27@hotmail.com (S.P. Arain), asifshahzadmmd@gmail.com (M.A. Shahzad), pharmacistscholar@gmail.com (E. Dar), dranila99@yahoo.com (A. Alam), alsherif.a@gmail.com (A.H.A. Hassanein).
} 
tie with accurate dose and time. ${ }^{8}$ In addition to this the proper duration of medicine therapy is also one of the important clinical aspect. ${ }^{9}$ The cost effectiveness should also be kept in mind especially when in the geriatrics poly pharmacy is suggested.Because if this factor is ignored especially in developing countries, it will lead to the inappropriate time duration of the therapy. ${ }^{10,11}$

Among the geriatrics patients the medicine consumption is more than one medicine hence it becomes very much problematic and challenging at the same time for the practitioner to achieve the optimum outcomes with less number of the medicines. Because as the number of medicine is more the more chances of the side effects and the drug/drug interaction complications. ${ }^{12,13}$ Standard protocols if adopted in recommending the medicine the quality of prescriptions can better be enhanced. Beers' criteria is the most authentic and frequently used tool to assess the appropriateness of the prescription. ${ }^{14}$

According to the Beers' criteria the irrational suggested medicines are categorized into these categories. Category A, Medicine which is avoided in the older adults Category B, Drug which crosses the maximum daily dose, Category C, Medicine which is averted in the particular condition of co morbidity. These are the Beers' criterion to suggest the medicine and if such recommendations are exercised in recommending medicine among geriatrics patients the vast number of complications can be subsided. ${ }^{15}$

The objective of the current study is to assess the irrational prescription of medicine among the geriatric patients in line with the Beers' criteria to evaluate the polypharmacy and point out the frequency of chronic disorders in geriatric patients.

\section{Methods}

It was cross sectional observational study conducted for the period of five months from November 2018 to April 2019 in a tertiary care Hospital of Larkana which is round about 1500 bed hospital and one of the biggest Hospital in the northern Sindh province. The Data was obtained from the case chart of the patients and the well developed questionnaire was administered among the patients. The patients were selected using the random sample techniques.Initially 550 questionnaires were distributed and in response 500 properly filled questionnaires were received and included in the study. The patients aged more than sixty years were mentally sound and upon showing consent were included in the study. Those patients who were mentally ill, required the intensive care unit (ICU) and/or when data was not available were excluded from the study. This study followed the Strengthening the Reporting of Observational Studies in Epidemiology (STROBE) Statement: guidelines for reporting observational studies. ${ }^{16}$

\subsection{Ethical approval}

The study was approved from the ethical committee of the Chandka Medical hospital Larkana, Pakistan. And the verbal consent was also sought from the patients after telling them all the details of the study.

\section{Results}

The patients demographic data was collected from the patients case charts which included the age wise, gender wise distribution, reasons for admission in the hospital and stay length in hospital as described in Table 1.

The most prescribed drugs were Antibiotics 25.4\% and NSAIDs 14.7\% between the 3108 prescribed medicines as illustrated in Table 2 .

Parenteral route of administration was observed in $70.04 \%$ of patients as demonstrated in Table 3.

The inappropriate medication use (IMU) was observed among 113 (22.6)\% patients. Inappropriate antibiotics were more detected in $35.39 \%$, and Benzodiazepine $28.32 \%$ as described in Table 4 and Table 5.
Table 1

Demographic Characteristics of the patients $(\mathrm{N}=500)$.

\begin{tabular}{lll}
\hline Characteristics & Number (\%) & Percentage \\
\hline Male & 213 & $42.6 \%$ \\
Female & 287 & $57.4 \%$ \\
Age in years & & \\
$60-65$ & 245 & $49.0 \%$ \\
$66-70$ & 139 & $27.8 \%$ \\
$71-75$ & 78 & $15.6 \%$ \\
$>76$ & 38 & $7.6 \%$ \\
Disease for hospital stay & & \\
Respiratory Disorder & 189 & $37.8 \%$ \\
GIT Disorders & 102 & $20.4 \%$ \\
CVS Disorders & 87 & $17.4 \%$ \\
Musculoskeletal Disorder & 47 & $9.4 \%$ \\
Infectious Diseases & 33 & $6.6 \%$ \\
Endocrine & 11 & $2.2 \%$ \\
Miscellaneous & 31 & $6.2 \%$ \\
Hospital Stay (in Days) & & \\
$0-5$ & 125 & $25 \%$ \\
6-10 & 197 & $39.4 \%$ \\
11-15 & 129 & $25.8 \%$ \\
$>15$ & 49 & $9.8 \%$ \\
\hline
\end{tabular}

CVS: cardiovascular system, GIT: gastrointestinal tract.

Table 2

Class of medicine prescribed (Total prescribed Medicines 3108).

\begin{tabular}{lll}
\hline Class of Medicine & Number (\%) & Percentage \\
\hline Antibiotics & 790 & $25.42 \%$ \\
NSAID & 458 & $14.74 \%$ \\
Drugs acting RTI & 449 & $14.45 \%$ \\
Drugs of GIT & 433 & $13.93 \%$ \\
Drugs of CNS & 421 & $13.55 \%$ \\
Drugs affecting Hematological System & 380 & $12.22 \%$ \\
Drugs OF CVS & 177 & $5.69 \%$ \\
Total & 3108 & 100
\end{tabular}

CNS: central nervous system, CVS: cardiovascular system, GIT: gastrointestinal tract, NSAID: non-steroidal anti-inflammatory drugs, RTI: Respiratory tract infection.

Table 3

Route of administration.

\begin{tabular}{lll}
\hline Routes of Administration & Number & Percentage \\
\hline Parenteral & 2177 & $70.05 \%$ \\
Oral & 931 & $29.95 \%$ \\
Total & 3108 & $100 \%$ \\
\hline
\end{tabular}

Table 4

Appropriate and inappropriate Drugs frequency.

\begin{tabular}{lll}
\hline Variable & Frequency & Percentage \\
\hline Inappropriate & 113 & $22.6 \%$ \\
Appropriate & 387 & $77.4 \%$ \\
Total & 500 & $100 \%$ \\
\hline
\end{tabular}

\section{Discussion}

The irrational and inappropriate usage of medicine is one of the high consideration problem worldwide which is not only responsible for the patients and his relatives to incur high burden of the therapy but also leading actor to further worse the health condition of the patient rather than improving that. Hence the current study focused the inappropriate use of medicines among the geriatric patients Poly pharmacy tools or the criteria including Beers' criterion, MAI and HEDIS were noticed to point out potentially inappropriate medications has certain restrictions of the tools and criteria in the everyday clinical 
Table 5

Frequency of inappropriate medicine in geriatric Patients based on BEERS' criterion.

\begin{tabular}{llll}
\hline Category & Name of drug & $\begin{array}{l}\text { Number of } \\
\text { patients }\end{array}$ & Frequency \\
\hline A & Antibiotics & 40 & 35.40 \\
& Benzodiazepine & 32 & 28.32 \\
& NSAID & 17 & 15.04 \\
& CVS drugs & 14 & 12.39 \\
B & Drugs exceeded the daily suggested & 08 & 7.08 \\
& dose & & 1.77 \\
& Drugs not to be given in Specific co & 02 & 100 \\
& morbidity & 113 & \\
\hline
\end{tabular}

NSAID: non-steroidal anti-inflammatory drugs, CVS: cardiovascular system.

setups were recognized. ${ }^{17}$

Beers' guidelines which has been used in the current study usually is the strong tool which provides the ample guidance to avoid the inappropriate medicines in the geriatrics patients. Category A tells that the medicines which must be avoided in the geriatric patients is one of the major inappropriate use of medication was noted in terms of nonsteroidal anti-inflammatory drugs (NSAIDs), benzodiazepines (BDZ) and antibiotics were seen inappropriate. It suggested the percentage is quite High which needs the address by the healthcare team. ${ }^{18}$

According to the Beers' guidelines the 5 or more than medicine in the single prescription is supposed to be the poly pharmacy and most of the prescription in the present study were seen with more than 5 medicines.which clearly indicated that the length of patient stay in the hospital will increase and the rest of increased medicines will be linked with lengthy hospital stay or the adverse effects of the inappropriate medications will need the further medicines in line with increasing the hospital stay days of the patients. ${ }^{19}$

The current study is inconsistent with the study of Juno et al., 2018, Hyderabad Pakistan who conducted a similar study but he concluded that $48.5 \%$ of patients got the inappropriate medication and did not include the category B and C of BEERS' guidelines. ${ }^{20}$ However, studies concluded that the antibiotics were the most frequently inappropriately suggested medicine similarly the current study stated that about $35 \%$ which is the highest inappropriately used medication. ${ }^{21,22}$

There is an increasing evidence of medicines related issues such as inappropriate prescribing among older people with co-morbidities and polypharmacy. ${ }^{23}$ Inappropriate prescribing is an important risk factor for adverse drug reactions and hospitalizations in the older people. ${ }^{24}$

Residents of nursing homes often suffer from many conditions that require the use of multiple drugs. A cross-sectional study of the longterm care population of six nursing homes examined the prevalence of inappropriate medications (PIMs) and their association with sex, age, number of drugs. They identified PIMs by applying the Norwegian General Practice Nursing Home (NORGEP-NH) standards. They analyzed the Poisson analysis to verify the relationship between the number of PIMs, sex, age, drug number and study location. They recognized PIMs in $56 \%$ of 103 geriatric residents (1.10 \pm 1.26$)$ with mean age 83 years and $68 \%$ women. The mean number of daily used medication is $(7.2 \pm 3.6)$. In the adjusted investigation, residents more than 80 years had $43 \%$ fewer PIMs matched with residents less than 80 years $(\mathrm{p}<0.05)$. Residents prescribed from 4 to more than 10 medications had on average 0.73 to 2.11 , more PIMs in comparison to residents given $\leq 3$ medicines ( $\mathrm{p}<0.001$ ), correspondingly. prevalence of inappropriate medications is common amongst nursing home people and is significantly connected with age and the polypharmacy. ${ }^{25}$

A systematic review of PIM-lists was conducted. The search strategy was run in databases from 1991 to 2017. All PIMs described in those lists were extracted and stratified by their potential cardiovascular (CV) risk counting major adverse CV events (MACE). The frequency of each PIM was documented and appraised. They detected 724 papers, and 24 were included. From those, a total of 21 drug-disease interactions were recovered and 17 PIMs to be avoided by the elderly. The documenting of PIMs with a hazard of CV adverse events was $15.3 \%$, whereas the documenting of those with MACE hazard was $7.2 \%$. The majority of described PIMs were 50\% tricyclic antidepressants, $45.8 \%$ centrally acting antiadrenergic agents, $29.2 \%$ NSAIDs, 25\% antiarrhythmics (Class I and III), 25\% peripherally acting antiadrenergic drugs and $20.8 \%$ antithrombotic agents. The mainly given PIMs with MACE hazards were $29.2 \%$ NSAIDs, $29.2 \%$ antiarrhythmics (Class I and III), 25\% selective calcium channel blockers with vascular effects and $16.7 \%$ antipsychotics. Data recommend that PIM-lists focus mostly on common adverse events and often inadequately illustrate the potential effect on MACE occurrence. ${ }^{26}$

A cross-sectional study was conducted using a convenience sample from four long-term care facilities and one community pharmacy in Portugal. They determined the prevalence of PIMs with a hazard of cardiac and cerebrovascular adverse events (CCVAEs), encompassing major adverse cardiac and cerebrovascular events (MACCE). A total of 680 elderly patients were involved. About 404 (59.4\%) patients were taking medicines connected with CCVAEs risk, involved 264 (38.8\%) patients who prescribed medications with MACCE risk. About $50 \%$ of patients with a previous record of CVD $(n=521)$ were taking PIMs with the hazard of CCVAEs, involving 30\% with the hazard of MACCE. Their results stated that $50 \%$ of patients with the previous record of CVD were prescribed medications with hazards of CCAVEs and $30 \%$ with hazards of MACCE. More tailored instruments for the management of drug treatment in elderly patients with CVD are of chief significance in clinical practice. ${ }^{27}$

The key outcome of a cross-sectional study was the percentage of hospitalized elderly patients taking at least one PIM at admission and discharge in Japan. PIMs were recognized depended on the 2015 American Geriatric Society Beers Criteria. The median number of medicines at admission was 5.0 (IQR 3.0-8.0). The percentages of patients taking any PIMs at admission and discharge were $47.9 \%$ (95\% CI: $0.44-0.52$ ) and $25.1 \%$ (95\% CI: $0.22-0.28$ ), correspondingly. The number of patients prescribed any PIMs was considerably lesser at discharge than at admission (Reduction rate [RR]: 0.48 , 95\% CI: 0.41-0.53). A significant part of hospitalized elderly patients with acute medical diseases prescribed PIMs at admission and discharge. These results should be established at other hospitals in Japan. ${ }^{28}$

Co-morbidity and polypharmacy of the elderly people increase the possibility of elderly in residential long-term care facilities experiencing inappropriate medication utilization (IMU). Databases (EMBASE, Google Scholar and MEDLINE) were mined for literature between 2004 and 2016 to recognize studies investigating IMU in long-term care services for the elderly. Studies were eligible when relying on ACOVE, Beers criteria, BEDNURS, PRISCUS, START, STOPP list, or MAI tools. Seventeen from 21 studies relied on a version of Beers criteria with predominance ranging from $18.5 \%$ to $82.6 \%$ (The median is $46.5 \%$ ) residents experiencing IMU. A smaller range, between $21.3 \%$ and $63.0 \%$ (The median is $35.1 \%$ ), was documented when considering exclusively the 10 studies that used Beers criteria updated in 2003. Predominance ranged between $23.7 \%$ and $79.8 \%$ (The median is $61.1 \%$ ) in seven studies based on STOPP. START and ACOVE were recognized in respectively four (Prevalence: $30.5 \%-74.0 \%$ ) and two studies (Prevalence: $28.9 \%-58.0 \%$ ); BEDNURS, MAI and PRISCUS were all applied in one study each. Beers criteria of 2003 and STOPP were mainly used to detect IMU in residential long-term care facilities. ${ }^{29}$

A systematical review estimated the dominance of potentially inappropriate medication use (PIMU) in nursing home elderly residents. The articles documented the results from 43 studies, of which 26 offered point prevalence estimation of PIMU (227,534 nursing home residents). The total weighted point predominance of PIMU in nursing homes was $43.2 \%$ (95\% CI: $0.37-0.49$ ), rising from $30.3 \%$ in studies conducted throughout $1990-1999$ to $49.8 \%$ in studies carried out after 2005 ( $\mathrm{P}<0.001)$. Point prevalence estimation documented in 
European countries was set up to be higher $49.0 \%$ (95\% CI: 43-56) than those documented in North America $26.8 \%$ (95\% CI: $16.5-37)$ or in other countries $29.8 \%$ (95\% CI: 19-40). Additionally, 18 studies representing 326,562 nursing home residents presented 20 area prevalence estimation ranging between $2.3 \%$ and $50.3 \%$. The overall number of prescribed medicines was consistently documented as the major leading aspect of PIMU. This systematic review stated that almost $50 \%$ of nursing home elderly residents are exposed to PIMU and recommends an enhanced predominance over time. Efficient interventions to optimize drug prescription in nursing home services are required. ${ }^{30-32}$

The current study encompasses a big sample size in a tertiary hospital. The study revealed the inappropriate medications among the elderly, in various therapy categories. The study is important to improve the safety of prescribing medications for the elderly. It emphasizes the deprescribing of medications that are unnecessary, which helps to reduce the problems of polypharmacy, drug interactions, and adverse drug reactions, thereby improving the risk-benefit ratio of medication regimens in at-risk people and increasing the rational medications use among elderly patients. On the other hand, the study had some limits. Firstly, the study was done in one site only which limits the generalization of findings. Secondly, the study's design was cross-sectional which not determine the association of risk factors nor causality. Finally. recall bias can happen if the study inquires contributors about the long-ago experience.

\subsection{Recommendation}

There is a dire need to place the clinical Pharmacist in action to play his very important role in subsiding the Drug/Drug interaction, to avoid the polypharmacy and emphasis the appropriate and rational use of medications among the geriatric patients.

\section{Conclusion}

Polypharmacy is highly prevalent in the prescriptions of the geriatrics patients, due to the certain reasons like chronic disease. Even though it is not easy to get rid of polypharmacy however the care should be exercise to suggest less number of medications with the optimum outputs. This study may help healthcare providers in the recognition and deprescribing of these medications in elderly patients with high risk during medications assessment.

\section{Funding}

This research did not receive any specific grant from funding agencies in the public, commercial, or not-for-profit sectors.

\section{IRB approval}

The study was approved from the ethical committee of the Chandka Medical hospital Larkana.

\section{Authors' contributions}

- AAM contributed in the conception of the work, assigned the Beer's score, data collection, complete was the data in retrospective chart review, data analysis, manuscript drafting, manuscript revising, and approval of manuscript final, all aspects of the work and agreed to all aspects of the study.

- MAH contributed to the conception of the work, data analysis, abstracted the data, manuscript revising, and manuscript final approval and agreed to all aspects of the study.

- HK contributed to the data collection, the conception of the work, manuscript revising, and manuscript final approval and agreed to all aspects of the study.
- SPA contributed in the manuscript drafting, manuscript revising, manuscript final approval and agreed to all aspects of the work.

- MAS contributed in the data collection, manuscript revising, manuscript final approval and agreed to all aspects of the work.

- ED contributed to the manuscript revising, and manuscript final approval and agreed to all aspects of the study.

- AA contributed in the conception of the work, data analysis, manuscript drafting, manuscript revising, and approval of manuscript final and agreed to all aspects of the work.

- AHAH contributed to the manuscript revising, and manuscript final approval and agreed to all aspects of the study.

The manuscript has been read and agreed by all the authors. The requirements for authorship as stated earlier in this document have been encountered. Each author deliberates that the manuscript represents honest work.

\section{Declaration of competing interest}

All the authors do not have any possible conflicts of interest.

\section{Acknowledgments}

Our thank is to all members of Hospital of Larkana Sindh Hospital of Larkana Sindh for their effective collaboration.

\section{References}

1. Knapowski J, Wieczorowska-Tobis K, Witowski J. Pathophysiology of ageing. $J$ Physiol Pharmacol. 2002 Jun;53:135-146 PMID: 12120891.

2. Wyss-Coray T. Ageing, neurodegeneration and brain rejuvenation. Nature. 2016 Nov 10;539:180-186. https://doi.org/10.1038/nature20411.

3. Adelnia F, Cameron D, Bergeron CM, et al. The role of muscle perfusion in the ageassociated decline of mitochondrial function in healthy individuals. Front Physiol. 2019 Apr 12;10:427. https://doi.org/10.3389/fphys.2019.00427.

4. Hammad MA, Syed Sulaiman SA, Aziz NA, et al. Prescribing statins among patients with type 2 diabetes: the clinical gap between the guidelines and practice. $J$ Res Med Sci. 2019 Feb 25;24:1-6. https://doi.org/10.4103/jrms.JRMS 10018.

5. Hammad MA, Khamis AA, Al- Akhali KM, et al. Evaluation of drug dosing in renal failure. IOSR-JPBS. 2016;11:39-50. https://doi.org/10.9790/3008-1105033950.

6. Hammad MA, Tangiisuran B, Kharshid AM, et al. Drug-drug interaction-related uncontrolled glycemia. J Pharm BioAllied Sci. 2017;9:221-228. https://doi.org/10. 4103/jpbs.JPBS_26_17.

7. Ullah Amman, Majid Khan Gul, ud-Din Fakhar. Inappropriate and irrational use of medicines prescribed to the geriatric patients in Pakistan. ARC J Pub Health Community Med. 2018;3:12-18. https://doi.org/10.20431/2456-0596.0302003.

8. World Health Organization (WHO). The pursuit of responsible use of medicines: sharing and learning from country experiences. Available at: https://www.who.int/ medicines/areas/rational_use/en/, Accessed date: 17 August 2019.

9. Nepal G, Bhatta S. Self-medication with antibiotics in WHO southeast Asian region: a systematic review. Cureus. 2018 Apr 5;10:e2428. https://doi.org/10.7759/cureus. 2428.

10. Hammad MA, Abdo MS, Mashaly AM, et al. The statins effects on HbA1c control among diabetic patients: an umbrella review of systematic reviews and meta-analyses of observational studies and clinical trials. Diabetes Metab Syndr: Clin Res Rev. 2019;13:2557-2564. https://doi.org/10.1016/j.dsx.2019.07.005.

11. Mboya EA, Sanga LA, Ngocho JS. Irrational use of antibiotics in the Moshi Municipality Northern Tanzania: a cross sectional study. Pan Afr Med J. 2018;31:165. https://doi.org/10.11604/pamj.2018.31.165.15991.

12. Mannucci PM, Nobili A, Pasina L, et al. Polypharmacy in older people: lessons from 10 years of experience with the REPOSI register. Intern Emerg Med. 2018 Dec;13:1191-1200. https://doi.org/10.1007/s11739-018-1941-8.

13. Hammad MA, Syed Sulaiman SA, Aziz NA, et al. Evaluation of statins impacts on cognitive function among diabetic patients. Diabetes Metab Synd: Clin Res Rev. 2019;13:1797-1803. https://doi.org/10.1016/j.dsx.2019.04.006.

14. Fialová D, Kummer I, Držaić M, et al. Ageism in medication use in older patients. In: Ayalon L, Tesch-Römer C, eds. Contemporary Perspectives on Ageism. International Perspectives on Aging. vol. 19. Cham: Springer; 2018.

15. Singh J. Evaluation of the appropriateness of prescribing in geriatric patients using Beers' criteria and Phadke's criteria and comparison thereof by Rima Shah and colleagues. J Pharmacol Pharmacother. 2012 Jan;3. https://doi.org/10.4103/0976500X.92509 8-2.

16. Enhancing the QUAlity and transparency of health research. Equator network. Available at: http://www.equator-network.org/reporting-guidelines/strobe/, Accessed date: 17 August 2019.

17. Mao W, $\mathrm{Vu} \mathrm{H}$, Xie Z, et al. Systematic review on irrational use of medicines in China and Vietnam. PloS One. 2015 Mar 20;10:e0117710https://doi.org/10.1371/journal. pone. 0117710 . 
18. Motter FR, Fritzen JS, Hilmer SN, et al. Potentially inappropriate medication in the elderly: a systematic review of validated explicit criteria. Eur J Clin Pharmacol. 2018 Jun;74:679-700. https://doi.org/10.1007/s00228-018-2446-0.

19. The 2019 American geriatrics society Beers Criteria ${ }^{\circledR}$ update expert panel American geriatrics society 2019 updated AGS Beers Criteria ${ }^{\circledR}$ for potentially inappropriate medication use in older adults. JAGS. April 2019;67:674-694. https://doi.org/10. 1111/jgs.15767.

20. Juno, Rahman N, Dayo A, et al. Evaluation of potentially inappropriate medications and polypharmacy among geriatrics in public hospitals of Hyderabad. Lat Am J Pharm. 2018;37:189-195http://www.latamjpharm.org/resumenes/37/1/LAJOP_37_ 1_1_28.pdf.

21. Machowska A, Stålsby Lundborg C. Drivers of irrational use of antibiotics in Europe. Int J Environ Res Publ Health. 2018 Dec 23;16:27. https://doi.org/10.3390/ ijerph16010027.

22. Chua K-P, Fischer MA, Linder JA. Appropriateness of outpatient antibiotic prescribing among privately insured US patients: ICD-10-CM based cross sectional study. BMJ. 2019;364:k5092. https://doi.org/10.1136/bmj.k5092.

23. Al Akhali K, Hammad MA, Ansari MA. Evaluation of prevalence and pattern of anemia- a hospital based study in Aseer province, Kingdom of Saudi Arabia. $J$ Exp Med Surg Res. 2013;2:32-35https://www.researchgate.net/publication/270337927 EVALUATION_OF_PREVALENCE_AND_PATTERN_OF_ANEMIA_-_A_HOSPITAL_ BASED_STUDY_IN_ASEER_PROVINCE_KINGDOM_OF_SAUDI_ARABIA_ INTRODUCTION.

24. Xing XX, Zhu C, Liang HY, et al. Associations between potentially inappropriate medications and adverse health outcomes in the elderly: a systematic review and meta-analysis. Ann Pharmacother. 2019 May 25. https://doi.org/10.1177/ 10600280198530691060028019853069.

25. Halvorsen $\mathrm{KH}$, Kucukcelik S, Garcia BH, et al. Assessing potentially inappropriate medications in nursing home residents by NORGEP-NH criteria. Pharmacy (Basel). 2019 Mar 5;7:26. https://doi.org/10.3390/pharmacy7010026.

26. Aguiar JP, Brito AM, Martins AP, et al. Potentially inappropriate medications with risk of cardiovascular adverse events in the elderly: a systematic review of tools addressing inappropriate prescribing. J Clin Pharm Therapeut. 2019 Jun;44:349-360. https://doi.org/10.1111/jcpt.12811.

27. Aguiar JP, Heitor Costa L, Alves da Costa F, et al. Identification of potentially inappropriate medications with risk of major adverse cardiac and cerebrovascular events among elderly patients in ambulatory setting and long-term care facilities. Clin Interv Aging. 2019 Mar 4;14:535-547. https://doi.org/10.2147/CIA.S192252.

28. Komagamine J. Prevalence of potentially inappropriate medications at admission and discharge among hospitalised elderly patients with acute medical illness at a single centre in Japan: a retrospective cross-sectional study. BMJ Open. $2018 \mathrm{Jul}$ 19;8:e021152https://doi.org/10.1136/bmjopen-2017-021152.

29. Storms H, Marquet K, Aertgeerts B, et al. Prevalence of inappropriate medication use in residential long-term care facilities for the elderly: a systematic review. Eur J Gen Pract. 2017 Dec;23:69-77. https://doi.org/10.1080/13814788.2017.1288211.

30. Morin L, Laroche ML, Texier G, et al. Prevalence of potentially inappropriate medication use in older adults living in nursing homes: a systematic review. J Am Med Dir Assoc. 2016 Sep 1;17:862. https://doi.org/10.1016/j.jamda.2016.06.011 e1-9.

31. Abdoa MS, Hammad MA, Noor Harun S, et al. Evaluation of Knowledge, Attitude and Practice of Healthcare Providers towards Life-Threatening Drug-Drug Interactions in Penang General Hospital. Malaysia: CEGH; 2020 April xxx-xxx. In press https://doi. org/10.1016/j.cegh.2020.04.023.

32. Hammad MA, Syed Sulaiman SA, Alghamdi S, et al. Statins-related peripheral neuropathy among diabetic patients. Diabetes Metab Syndr: Clin Res Rev. 2020;14(4):341-346. https://doi.org/10.1016/j.dsx.2020.04.005. 